\title{
Pressure-Induced Electronic and Structural Phase Evolution in the van der Waals Compound $\mathrm{FePS}_{3}$
}

\author{
C. R. S. Haines* \\ Cavendish Laboratory, Cambridge University, J.J. Thomson Ave, Cambridge CB3 OHE, United Kingdom \\ and Department of Earth Sciences, Cambridge University, Downing Street, Cambridge CB2 3EQ, United Kingdom \\ M. J. Coak ${ }^{\dagger}$ \\ Cavendish Laboratory, Cambridge University, J.J. Thomson Ave, Cambridge CB3 OHE, United Kingdom, \\ Center for Correlated Electron Systems, Institute for Basic Science, Seoul 08826, Republic of Korea, \\ and Department of Physics and Astronomy, Seoul National University, \\ Seoul 08826, Republic of Korea \\ A. R. Wildes \\ Institut Laue-Langevin, 71 Avenue des Martyrs, 38042 Grenoble Cedex 9, France \\ G. I. Lampronti \\ Department of Earth Sciences, Cambridge University, Downing Street, Cambridge CB2 3EQ, United Kingdom \\ C. Liu and P. Nahai-Williamson \\ Cavendish Laboratory, Cambridge University, J.J. Thomson Ave, Cambridge CB3 OHE, United Kingdom \\ H. Hamidov \\ Cavendish Laboratory, Cambridge University, J.J. Thomson Ave, Cambridge CB3 OHE, United Kingdom, \\ Navoiy Branch of the Academy of Sciences of Uzbekistan, Galaba Avenue, Navoiy, Uzbekistan, \\ and National University of Science and Technology "MISiS," \\ Leninsky Prospekt 4, Moscow 119049, Russia \\ D. Daisenberger \\ Diamond Light Source, Chilton, Didcot OX11 ODE, United Kingdom \\ S. S. Saxena \\ Cavendish Laboratory, Cambridge University, J.J. Thomson Ave, Cambridge CB3 OHE, United Kingdom \\ and National University of Science and Technology "MISiS," Leninsky Prospekt 4, \\ Moscow 119049, Russia
}

(Received 17 April 2018; published 28 December 2018)

\begin{abstract}
Two-dimensional materials have proven to be a prolific breeding ground of new and unstudied forms of magnetism and unusual metallic states, particularly when tuned between their insulating and metallic phases. Here we present work on a new metal-to-insulator transition system $\mathrm{FePS}_{3}$. This compound is a two-dimensional van der Waals antiferromagnetic Mott insulator. We report the discovery of an insulatormetal transition in $\mathrm{FePS}_{3}$, as evidenced by x-ray diffraction and electrical transport measurements, using high pressure as a tuning parameter. Two structural phase transitions are observed in the x-ray diffraction data as a function of pressure, and resistivity measurements show evidence of the onset of a metallic state at high pressures. We propose models for the two new structures that can successfully explain the x-ray diffraction patterns.
\end{abstract}

DOI: 10.1103/PhysRevLett.121.266801

Introduction.-Recent and substantial interest in $\mathrm{FePS}_{3}$ stems from the fact that, like graphite, $\mathrm{FePS}_{3}$ can be delaminated to give single layers. Its magnetic properties, and particularly the Ising-like nature of its Hamiltonian, make the compound particularly promising as it maintains its bulklike magnetic behavior even for a single monolayer [1]. As the $a b$ planes of the metal ions are linked only by van der Waals forces, the $M \mathrm{P} X_{3}$ family form model two-dimensional antiferromagnetic systems, leading to a number of more recent publications on the magnetic 
structures [2-6] and even attracting the moniker "magnetic graphene" [7].

The family of compounds here designated $M \mathrm{P} X_{3}$, where $M$ represents a transition metal such as $\mathrm{Fe}, \mathrm{Ni}$, or $\mathrm{Mn}$, and $X=\mathrm{S}$ or $\mathrm{Se}$, are easily synthesized and have been widely categorized, historically due to interest in lithium intercalation between crystal planes to serve as a battery material [8-18]. A comprehensive review is given by Grasso and Silipigni [19]. These materials all have very similar crystal structures and interactions, but subtly differing magnetic properties across the metal elements due to the richness of the exchange couplings present. Crystallographic data from $\mathrm{x}$-ray diffraction studies $[10,12]$ give a monoclinic unit cell with space group of $C 2 / \mathrm{m}$ and a honeycomb arrangement of the magnetic metal ions.

The $M \mathrm{P} X_{3}$ compounds are all $p$-type semiconductors [19] with extremely high room-temperature resistivities (reflecting the high purity of samples that can be produced) and band gaps in excess of $1 \mathrm{eV}$ as determined by optical measurements [20]. A series of calculations of the band structure have been carried out [17,21-23] but do not successfully reproduce the insulating state of these materials, predicting half-filled metallic bands. This leads to the conclusions that $M \mathrm{P} X_{3}$ are Mott insulators, and as such could be driven to an insulator-metal or Mott transition by applying pressure to tune the band structure. $\mathrm{FePS}_{3}$ was chosen to investigate as it has the lowest resistivity and band gap of the easily synthesized compounds and was therefore assumed to require the least pressure to metallize. Initial evidence of metallic behavior was first presented at the SCES2014 conference by Haines et al. [24] and then again independently by Tsurubayashi et al. [25]. This Letter is the first published report detailing the metalinsulator transition in $\mathrm{FePS}_{3}$.

Substantial interest lies in tuning Mott insulators towards their metallization transition. The simple Hubbard model traditionally used to describe such systems only yields solutions in the limiting metallic and insulating cases. Tuning the parameters of the system to an intermediate state accesses physics that is not yet fully understood. Additionally, many unconventional superconducting materials are low dimensional and lie in close proximity to antiferromagnetic Mott insulator phases in their phase diagrams, and theoretical calculations [26] suggest that these states have a strengthening influence on the formation of superconductivity. Tunable (for instance, through pressure) antiferromagnetic two-dimensional Mott insulators then provide a rich and clean environment to probe the core mechanisms of several unsolved problems in condensed matter physics.

Methods.-High-pressure XRD patterns were collected at room temperature at the Diamond Light Source on the I15 beam line. $\mathrm{FePS}_{3}$ is difficult to grind into an isotropic powder. This is due to the fact that when put under strain the crystals tend to shear parallel to the $a b$ planes leading to small platelets and therefore strong preferred orientation. In an attempt to mitigate this the samples were ground in liquid nitrogen. The diamond anvil cell was filled with powdered sample. No pressure transmitting medium was used. X rays with an energy of $29.2 \mathrm{keV}(\lambda=0.4246 \AA)$ were used to collect the diffraction patterns. This is sufficiently high energy to pass through the diamond anvils. A MAR345 2D detector was used to record the diffraction pattern with exposure times of between 15 and $45 \mathrm{~s}$. Pressure in the diamond anvil cell was determined by measuring the shift in the fluorescence wavelength of ruby [27] spheres that were placed inside the high pressure region. The data were initially processed using DAWn [28] (with a $\mathrm{LaB}_{6}$ calibration) and the subsequent Rietveld refinements were calculated using TOPAS-Academic [29].

Measurements of resistance were performed using a Keithley 2410 source meter with a fixed supplied current of $0.01 \mu \mathrm{A}$ and verified on a Keithley electrometer at $40 \mathrm{~V}$. The samples measured were rectangular single crystals and a four-wire Kelvin method was used to calculate resistivity from the measured resistance. As the current-voltage response of this sample is not simply Ohmic, the same excitation was used throughout to allow comparison of data-but limiting the measurable range of resistances. Contact resistances to $\mathrm{FePS}_{3}$ were found to be significant if precautions were not taken - on order of $\mathrm{M} \Omega$ for simple silver epoxy electrical contacts, and with significant voltage dependence. To avoid this, the ambient pressure measurements were prepared with gold conducting pads evaporated onto the sample surface. In the Bridgman cells used for the high-pressure resistivity measurements, contact is formed by mechanically pressing Pt wires into the sample, which was seen to give good contact.

Measurements were performed in several Bridgman anvil cells [30] with both tungsten carbide and sintered diamond anvils. A steatite powder pressure medium was used in the Bridgman cells, leading to a uniaxial component (along the sample $c *$ axis) of the pressure achieved, as well as pressure gradients within the sample estimated at around $20 \%$. The pressure in these cells is estimated from the load applied during pressurization.

Temperature control was achieved through an Oxford Instruments Heliox He-3 cryostat, an ICE Oxford $1 \mathrm{~K}$ pumped helium cryostat, and an adiabatic demagnetization refrigerator developed in house. Care was taken to vary temperatures slowly (typically a few kelvin per hour) to allow the large thermal mass of the pressure cells to equilibrate.

Results. - It was clear upon observing the evolution of the diffraction patterns, see Fig. 1, that there were two phase transitions. The first phase transition (PT1) at approximately $4 \mathrm{GPa}$ and the second (PT2) at approximately $14 \mathrm{GPa}$. The structure is known at ambient pressure (0 GPa); however, the high-pressure structures were not known. The first task was therefore to produce physically 


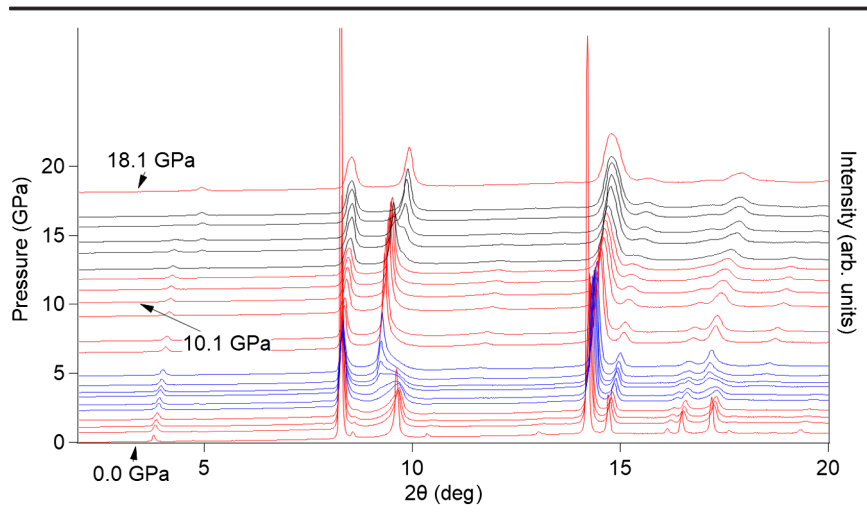

FIG. 1. Integrated diffraction patterns. The data have been scaled to the low angle background (giving arbitrary intensity, see right $y$ axis) and then the offset set to the pressure at which the data were collected (shown on the left $y$ axis). The 0.0 GPa data have been truncated for the two highest intensity peaks to allow all the patterns to be plotted together. The two phase transitions can be seen to take place over the region colored blue (PT1) and that colored black (PT2). The three patterns that we identified as being monophase are labeled with their pressures. The wavelength of the $\mathrm{x}$ rays was $\lambda=0.4246 \AA$.

sensible models for the two unknown structures that could be used to fit the high-pressure diffraction patterns. In both phase transitions new diffraction peaks develop while other peaks disappear. This allowed the identification of monophasic diffraction patterns for all three phases: the pattern at $0 \mathrm{GPa}$ corresponds to the low-pressure (ambient pressure) monoclinic $C 2 / \mathrm{m}$ phase $[10,12]$; the one at $10.1 \mathrm{GPa}$ to a high-pressure phase, here designated HP I; finally, the pattern at $18.1 \mathrm{GPa}$ corresponds to a second high-pressure phase, HP II. Assuming only that the structure does not go through a major reconstruction, we can equate the lowest angle (highest $d$ spacing) peak at around a $2 \theta$ of $3^{\circ}-4^{\circ}$ in Fig. 1 to the interplanar distance. The details of the refinements can be found in the Supplemental Material [31].

Figure 2 shows views of the refined structures for the different phases. At ambient pressure, the sulfur atoms form two-dimensional hexagonally coordinated layers, and there is a close-packing coordination between the layers. The close packing is not visible in the view along the $c *$ axis featuring all the atoms [Fig. 2(b) for HP 0] because sulfur atoms are directly below $\mathrm{Fe}$ and $\mathrm{P}$ atoms, but is visible if only the sulfur atoms are shown [Fig. 2(c) for HP 0]. A shear of $\sim a / 3$ along the $a$ axis, however, preserves the close packing between the two sulfur layers while reducing the volume of the unit cell by putting the Fe atoms directly above one another, and likewise for the $\mathrm{P}$ atoms. This is apparent in the view along $c *$, shown in Fig. 2. The HP I structure also appears to show some buckling of the sulfur layers, possibly due to strain. The buckling disappears at the higher-pressure transition to HP II, where the structure adopts a higher symmetry. The pressure dependence of the interplanar distance is shown in Fig. 2(a). At the second

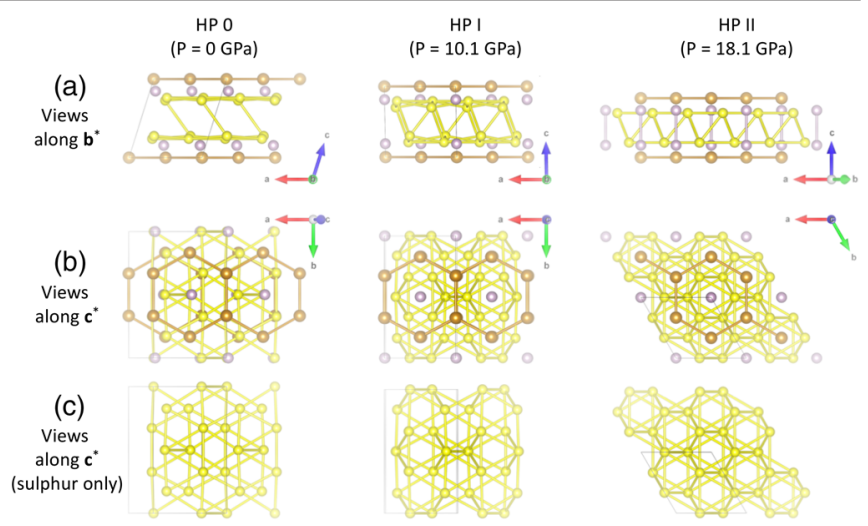

FIG. 2. Schematics showing the evolution of the structure of $\mathrm{FePS}_{3}$ with pressure. The three refined structures at their corresponding pressures are drawn to the same scale and with respect to the lattice parameters for the structures given in Tables S2-S4 in the Supplemental Material [31]. The Fe atoms are shown in brown, the $\mathrm{P}$ atoms are shown in purple, and the $\mathrm{S}$ atoms are shown in yellow. The views show different projections of the same number of unit cells; hence the "sulfur" only figures show only those sulfurs between two adjacent $a b$ planes. Also shown are all interatomic bonds for $r \leq 3.6 \AA$. The illustrations were created using the VESTA software [37].

phase transition there is a dramatic collapse of the interplanar distance: from approximately 5.7 to $4.9 \AA$. This is a collapse of nearly $15 \%$. More detail can be found in the Supplemental Material [31]. The Supplemental Material also includes a description of the pressure dependence of the lattice parameters of the three phases identified.

The temperature dependence of the resistivity $\rho$ of $\mathrm{FePS}_{3}$ in the absence of any applied pressure is plotted in Fig. 3, from room temperature down to the temperature when the resistance becomes too high to measure on the apparatus

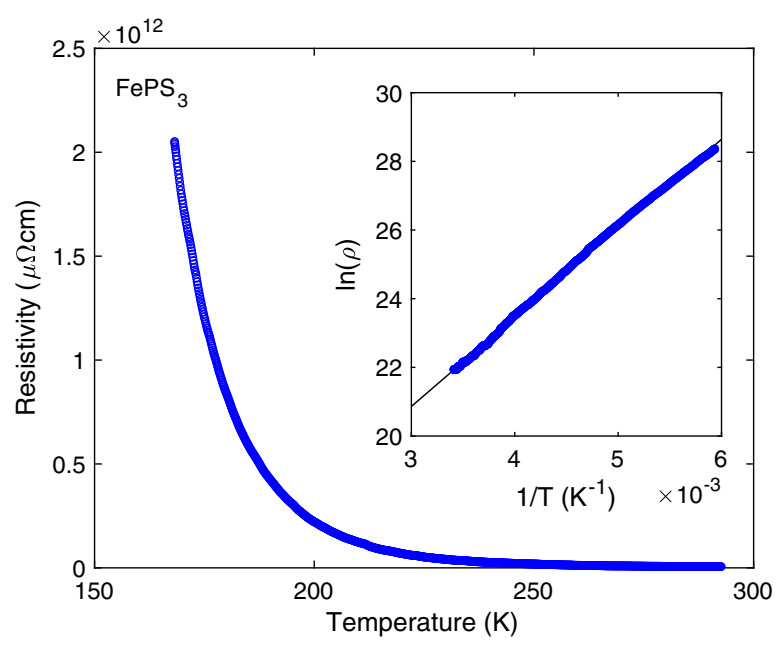

FIG. 3. Resistivity $\rho$ of $\mathrm{FePS}_{3}$ plotted against temperature at ambient pressure. The inset plots $\ln (\rho)$ against the reciprocal of temperature, showing good agreement with thermally activated Arrhenius-type behavior. 

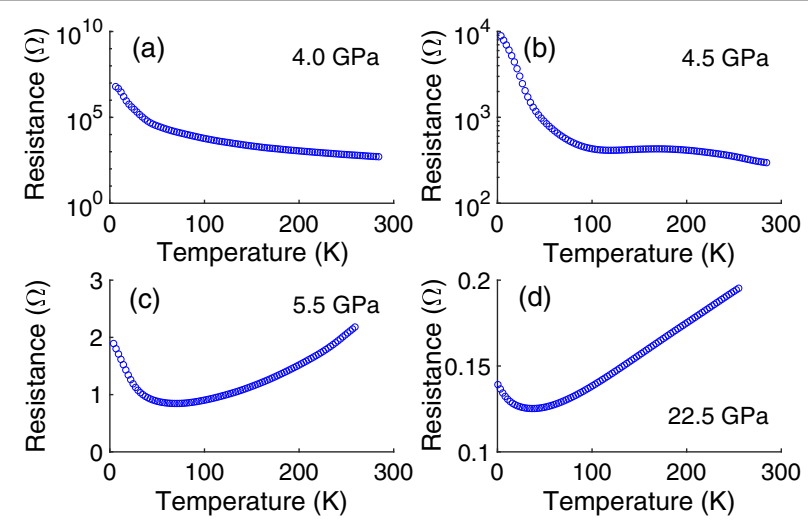

FIG. 4. Resistance of $\mathrm{FePS}_{3}$ against temperature for 4 increasing pressures, estimated as (a) $4.0 \mathrm{GPa}$, (b) $4.5 \mathrm{GPa}$, (c) $5.5 \mathrm{GPa}$, and (d) $22.5 \mathrm{GPa}$. A transition from insulating to metallic behavior is seen as pressure is increased, as well as an upturn in the resistivity at low temperatures in the high-pressure measurements.

used. The resistivity values found match with the orders of magnitude observed previously [13] and are insulating in nature. The inset shows a fit of the resistivity to an Arrhenius-type thermally activated conduction process across a fixed band gap $\rho \propto e^{T_{0} / T}$ - plotting $\ln \rho$ against $1 / T$ will yield a straight line if this relation holds, which is indeed seen in the graph, with some divergence towards the highest temperatures measured, potentially due to carrier saturation. The extracted band gap, found from the gradient and $T_{0}$, is then $0.452(1) \mathrm{eV}$. The overall result matches that in previous studies [38], but in this reference an activation energy of $0.60(1) \mathrm{eV}$ is quoted, and values up to $1.5 \mathrm{eV}$ have been found from optical measurements [8]. The different energy gaps obtained from the different measurements are explained by the fact that the optical absorption spectra and the conductivity measurements are sensitive to excitations generated in different bands. Both measurements are correct and there is no evidence for disorder or impurities playing a significant role on the values of energy obtained. See Grasso et al. for a full discussion [38].

Two independent sets of measurements in the quasihydrostatic Bridgman anvil cells are shown in Figs. 4 and 5. In both cases the stated pressure values are estimates based on the external load applied to the cells in pressurization, with a typical error of up to $20 \%$. The lower-pressure data [Fig. 4(a) and upper curves in Fig. 5] still show the activated behavior of an insulator, similar to the ambient pressure data, but increasing pressure drastically decreases the magnitude of the resistivity. At pressures above an estimated 5.0 GPa the resistivity begins to decrease with decreasing temperature-indicative of metallic behavior. The application of pressure also leads to a decrease in the magnitude of the resistivity over the measured temperature range by up to 8 orders of magnitude. Figure 4 also appears to capture an intermediate state, where the insulator-metal transition is visible as a broad peak in the resistance around

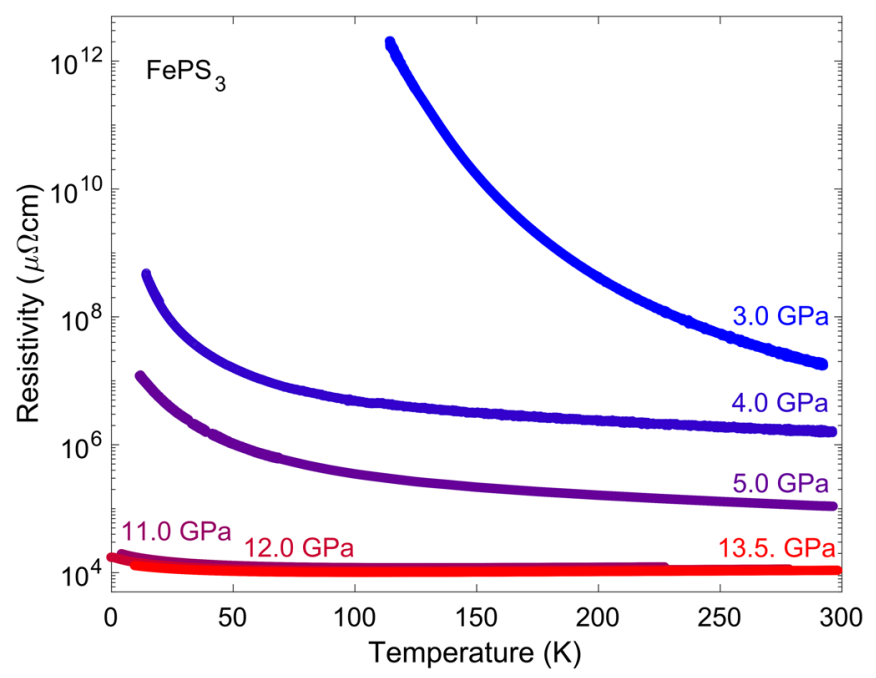

FIG. 5. Resistivity of $\mathrm{FePS}_{3}$ plotted against temperature, at pressures from an estimated 3.0 $\mathrm{GPa}$ (blue, topmost) to $13.5 \mathrm{GPa}$ (red) in a Bridgman anvil cell-reproducing the data shown in Fig. 4. The resistivity is drastically suppressed with applied pressure - note the logarithmic axis, and an upturn seen in the higher-pressure data.

$180 \mathrm{~K}$, but has moved outside of the measurable temperature range in subsequent pressure points.

In the higher-pressure data taken in Bridgman cells, shown in Figs. 4(c), 4(d), and 6, metallic (though sublinear-perhaps semimetallic) resistivity is observed at high temperatures. At lower temperatures, however, an upturn in the resistivity is seen. This feature should not be attributed to a simple insulating state, as the increase in resistivity as temperature decreases is slight and far from matching the exponential dependence of an Arrhenius or variable-range-hopping

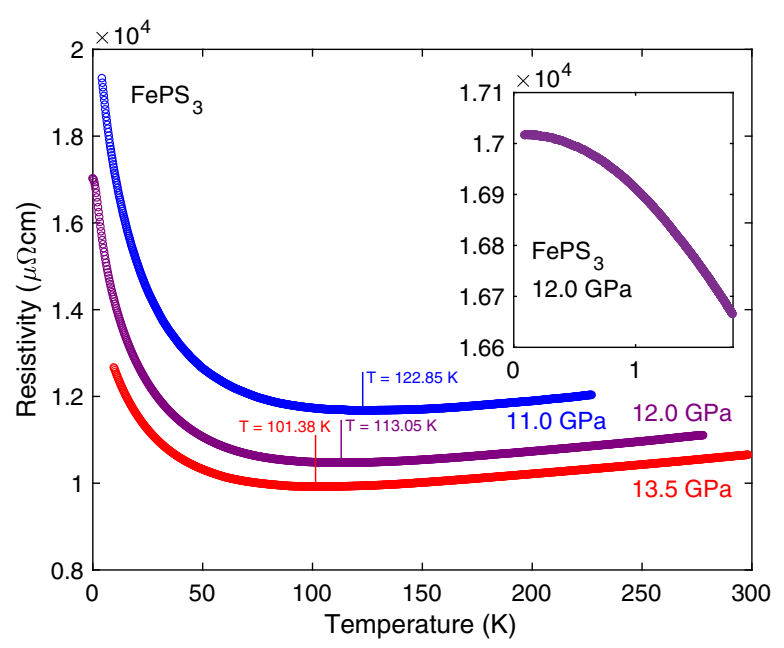

FIG. 6. Detail of Fig. 5. Resistivity of $\mathrm{FePS}_{3}$ plotted against temperature, at pressures from an estimated 11.0 GPa (blue, topmost) to $13.5 \mathrm{GPa}$ (red) in a Bridgman anvil cell. Inset: Lowtemperature data at $12.0 \mathrm{GPa}$, which levels off or saturates at the lowest temperatures. 
activated behavior, or the logarithmic dependence of the Kondo effect. The 12.0 GPa data, which were taken down to $100 \mathrm{mK}$ (Fig. 6, inset), additionally show a leveling off or saturation of the resistivity as the temperature approaches zero; this resembles the saturation effects commonly seen in semiconductors. What can however be concluded from Fig. 5 is that increasing the applied pressure suppresses both the temperature of the minimum and the magnitude of the upturn at the lowest temperature measured. $\mathrm{FePS}_{3}$ at ambient pressure orders antiferromagnetically at $120 \mathrm{~K}$, and it is tempting to speculate that the upturn may also have some connection to the magnetic properties of the compound at high pressure.

Discussion.-The application of pressure changes the structure and electronic properties of $\mathrm{FePS}_{3}$. Through the analysis of powder $\mathrm{x}$-ray diffraction data under pressure we have discovered and produced models for two previously unknown phases of $\mathrm{FePS}_{3}$. The change from the ambient pressure structure to HP I amounts to a shear of the unit cell by $\sim a / 3$ along the $a$ axis. A shear between the $a b$ planes with pressure seems feasible as the planes are only weakly bound by van der Waals forces and they slide easily over one another. Inspection of the stacking normal to the $a b$ planes, i.e., along the $c *$ axis, for the different structures supports a coherent shear model. Our models for the highpressure structures could form the starting point for a band structural calculation study aimed at identifying the precise mechanism for metallization.

Under ambient conditions, the layered van der Waals antiferromagnet $\mathrm{FePS}_{3}$ displays insulating behavior, with an electronic band gap around $0.5 \mathrm{eV}$, in agreement with previous studies. The application of comparatively low pressures, up to $2.0 \mathrm{GPa}$, has little effect on the resistivity but higher pressures reduce the sample's resistivity values at a dramatic rate. In the quasihydrostatic Bridgman cells, a reduction in sample resistance values from $\mathrm{G} \Omega$ to $\Omega$ ranges is seen, as well as the resistance increasing with temperature from pressures around 5.0 GPa, indicative of a metallic rather than insulating state. Electronic structure calculations suggest $\mathrm{FePS}_{3}$ to be a Mott or charge-transfer insulator, and this insulator-metal transition observed through applied pressure strongly supports this view. Additionally, in the metallic phase at pressures above the transition, an upturn in the resistivity is observed at temperatures below $150 \mathrm{~K}$, suppressed with increasing pressure. The upturn could not be described by any common forms of exponential insulatortype temperature dependencies or scattering mechanisms such as the Kondo effect. Further information on the band structure and nature of carriers in this high-pressure state is needed to properly understand this feature.

We show that $\mathrm{FePS}_{3}$ undergoes an insulator-metal transition at high pressures and we are able to correlate the electronic transition with concomitant changes in the crystallographic structure. The experiments are challenging, but the data are unambiguous and conclusive.
A strain- or pressure-induced transition to a metallic phase is not only relevant for the graphene community. The transition of a layered antiferromagnetic insulator to a conductor is also found in high-temperature superconductors, which is a subject of long-standing interest. The electronic properties of high-temperature superconductors are controlled by doping, and it is very difficult to unravel the effects that come into play in nonstoichiometric systems. Our study suggests another avenue, as the application of pressure to a stoichiometric system provides causal relations that are far less ambiguous, and we believe that pressure studies on $\mathrm{FePS}_{3}$ may also provide insight into the origin of high-temperature superconductivity.

This work was carried out with the support of the Diamond Light Source and we thank Heribert Wilhelm for advice and help with the x-ray diffraction experiments at Diamond. The authors would like to thank G. G. Lonzarich, P. A. C. Brown, S. E. Dutton, L. J. Spalek, and D. Jarvis for their help and discussions. We would also like to acknowledge support from Jesus College of the University of Cambridge, the Engineering and Physical Sciences Research Council (Doctoral Training Grant for M. J.C.), IHT KAZATOMPROM, and the CHT Uzbekistan program. The work was carried out with financial support from the Ministry of Education and Science of the Russian Federation in the framework of Increase Competitiveness Program of NUST MISiS (No. 2-2017-024). This work was supported by IBS-R009-G1.

*Corresponding author.

crsh2@cam.ac.uk

Corresponding author.

mattcoak@snu.ac.kr

Corresponding author. sss21@cam.ac.uk

[1] J. Lee, S. Lee, J. Ryoo, S. Kang, T. Kim, P. Kim, C. Park, J. Park, and H. Cheong, Nano Lett. 16, 7433 (2016).

[2] D. Lançon, H. C. Walker, E. Ressouche, B. Ouladdiaf, K. C. Rule, G. J. McIntyre, T. J. Hicks, H. M. Rønnow, and A. R. Wildes, Phys. Rev. B 94, 214407 (2016).

[3] A. R. Wildes, V. Simonet, E. Ressouche, G. J. McIntyre, M. Avdeev, E. Suard, S. A. J. Kimber, D. Lançon, G. Pepe, B. Moubaraki et al., Phys. Rev. B 92, 224408 (2015).

[4] A. Wildes, K. Rule, R. Bewley, M. Enderle, and T. Hicks, J. Phys. Condens. Matter 24, 416004 (2012).

[5] A. R. Wildes, H. M. Rønnow, B. Roessli, M. J. Harris, and K. W. Godfrey, Phys. Rev. B 74, 094422 (2006).

[6] A. Wildes, B. Roessli, B. Lebech, and K. Godfrey, J. Phys. Condens. Matter 10, 6417 (1998).

[7] J.-G. Park, J. Phys. Condens. Matter 28, 301001 (2016).

[8] R. Brec, D. M. Schleich, G. Ouvrard, A. Louisy, and J. Rouxel, Inorg. Chem. 18, 1814 (1979).

[9] K. C. Rule, G. J. McIntyre, S. J. Kennedy, and T. J. Hicks, Phys. Rev. B 76, 134402 (2007).

[10] G. Ouvrard, R. Brec, and J. Rouxel, Mater. Res. Bull. 20, 1181 (1985). 
[11] K. Kurosawa, S. Saito, and Y. Yamaguchi, J. Phys. Soc. Jpn. 52, 3919 (1983).

[12] W. Klingen, G. Eulenberger, and H. Hahn, Die Naturwissenschaften 55, 229 (1968).

[13] K. Ichimura and M. Sano, Synth. Met. 45, 203 (1991).

[14] P. A. Joy and S. Vasudevan, Phys. Rev. B 46, 5425 (1992).

[15] G. Le Flem, R. Brec, G. Ouvard, A. Louisy, and P. Segransan, J. Phys. Chem. Solids 43, 455 (1982).

[16] J. Rouxel, P. Molinie, and L. Top, J. Power Sources 9, 345 (1983).

[17] N. Kurita and K. Nakao, J. Phys. Soc. Jpn. 58, 610 (1989).

[18] N. Kurita and K. Nakao, J. Phys. Soc. Jpn. 58, 232 (1989).

[19] V. Grasso and L. Silipigni, Riv. Nuovo Cimento 25, 1 (2002).

[20] V. Grasso, S. Santangelo, and M. Piacentini, Solid State Ionics 20, 9 (1986).

[21] M. Whangbo, R. Brec, G. Ouvrard, and J. Rouxel, Inorg. Chem. 24, 2459 (1985).

[22] V. Zhukov, S. Alvarez, and D. Novikov, J. Phys. Chem. Solids 57, 647 (1996).

[23] M. Piacentini, F. Khumalo, C. Olson, J. Anderegg, and D. Lynch, Chem. Phys. 65, 289 (1982).

[24] C. R. S. Haines, M. J. Coak, P. Nahai-Williamson, C. Liu, and S. S. Saxena, in Proceedings of SCES2014, Grenoble, France, We-217 (2014), p. 788.

[25] M. Tsurubayashi, K. Kodama, M. Kano, K. Ishigaki, Y. Uwatoko, T. Watanabe, K. Takase, and Y. Takano, Pressure Dependence of Electric Resistivity of $\mathrm{FePS}_{3}$, Nihon University Science and Engineering Office, Academic Lecture Abstract Collection.
[26] P. Monthoux and G. G. Lonzarich, Phys. Rev. B 66, 224504 (2002).

[27] H. Mao, J. Xu, and P. Bell, J. Geophys. Res. 91, 4673 (1986).

[28] J. Filik, A. W. Ashton, P. C. Y. Chang, P. A. Chater, S. J. Day, M. Drakopoulos, M. W. Gerring, M. L. Hart, O. V. Magdysyuk, S. Michalik, A. Smith, C. C. Tang, N. J. Terrill, M. T. Wharmby, and H. Wilhelm, J. Appl. Crystallogr. 50, 959 (2017).

[29] A. A. Coelho, J. Appl. Cryst. 51, 210 (2018).

[30] J. Wittig, Z. Phys. 195, 215 (1966).

[31] See Supplemental Material at http://link.aps.org/ supplemental/10.1103/PhysRevLett.121.266801 for details of the space group assignment procedure for the two new phases, HP I and HP II, as well as the pressure evolution of the lattice parameters through the phase transitions, which includes Refs. [32-36].

[32] R. Allmann and R. Hinek, Acta Crystallogr. Sect. A 63, 412 (2007).

[33] P. W. Stephens, J. Appl. Crystallogr. 32, 281 (1999).

[34] A. Boultif and D. Louër, J. Appl. Crystallogr. 37, 724 (2004).

[35] W. I. F. David, K. Shankland, J. van de Streek, E. Pidcock, W. D. S. Motherwell, and J. C. Cole, J. Appl. Crystallogr. 39, 910 (2006).

[36] C. R. Groom, I. J. Bruno, M. P. Lightfoot, and S. C. Ward, Acta Crystallogr. Sect. B 72, 171 (2016).

[37] K. Momma and F. Izumi, J. Appl. Crystallogr. 44, 1272 (2011).

[38] V. Grasso, F. Neri, S. Patane, L. Silipigni, and M. Piacentini, Phys. Rev. B 42, 1690 (1990). 